\title{
Coccidies et coccidioses intestinales de la chèvre du Sahel (Hircus reversus) au Sénégal avec la description d'une espèce nouvelle
}

\author{
A. Maho ${ }^{1}$ B.S. Toguebaye ${ }^{1 *}$ J. Belot ${ }^{2}$
}

Mots-clés

Caprin - Chèvre du Sahel - Coccidia Coccidiose - Eimeria panguii n. sp. Espèce nouvelle - Sénégal.

\begin{abstract}
Résumé
Huit espèces d'Eimeria, dont une nouvelle, ont été trouvées chez la chèvre du Sahel (H ircus reversus) au Sénégal : E. arloingi, E. kocharli, E. alijevi, E. ninakohlyakimovae, E. christenseni, E. hirci, E. caprovina et Eimeria panguii $\mathrm{n}$. sp. Les oocystes d'Eimeria panguii $\mathrm{n}$. sp. étaient ovoïdes et dépourvus de capsule micropylaire. Ils mesuraient 48,19 $\pm 0,73$ x 32,45 $\pm 0,97 \mu \mathrm{m}(45-50 \times 29$ $36 \mu \mathrm{m})$. Le micropyle était étroit. Les résidus oocystiques étaient présents. Les sporocystes étaient ovoïdes, 16,86 $\pm 0,77$ x 8,93 $\pm 0,34 \mu \mathrm{m}(15-18 \times 8-10$ $\mu \mathrm{m})$. Le corps de Stieda était absent tandis que les résidus sporocystiques étaient présents. Les deux sporozoïtes étaient falciformes et inversés l'un par rapport à l'autre et se recouvraient par leurs deux extrémités. La transmission expérimentale de ces espèces à un chevreau a permis de mettre en évidence I'hypertrophie des cellules de l'épithélium intestinal, la perforation de la muqueuse intestinale et la chute de ses microvillosités.
\end{abstract}

\section{INTRODUCTION}

L'élevage intensif des petits ruminants peut être considéré comme la voie la plus rapide et la plus directe pour promouvoir le développement de l'élevage en Afrique ; mais l'efficacité d'un tel système demande la maîtrise de certains facteurs comme la santé. Parmi les problèmes sanitaires, il faut noter que la coccidiose est l'une des maladies qui constituent un frein au développement de la production intensive de moutons et de chèvres (14).

Dans le présent travail, les coccidies intestinales de la chèvre du Sahel au Sénégal sont décrites ainsi que les lésions qu'elles causent au niveau intestinal.

1. Laboratoire de Parasitologie, Département de Biologie Animale, Faculté des Sciences, Université Ch. A. Diop de Dakar, Dakar, Sénégal

2. Département de Parasitologie, Ecole Inter-Etat des Sciences et Médecine Vétérinaire, Dakar, Sénégal

* Adresse pour la correspondance : Faculté des Sciences et Techniques, Dakar, Sénégal

Fax : (221) 246318 ou 252529
MATERIEL ET METHODES

\section{Récolte d'oocystes dans les matières fécales}

Les oocystes de coccidies ont été obtenus à partir des matières fécales prélevées dans le rectum des chèvres du Sahel abattues aux abattoirs de Dakar. Quatre-vingt-cinq prélèvements ont été effectués et soumis à des analyses coproscopiques pour rechercher les coccidies. Cinquante de ces prélèvements se sont révélés positifs.

L'opération de concentration des oocystes a ensuite été effectuée. Vingt grammes de matière fécale des prélèvements positifs ont été triturés et versés dans $80 \mathrm{ml}$ de solution d'eau saturée de sel de cuisine. Après homogénéisation, la suspension a été tamisée et versée dans une boîte de Pétri. Un plastique transparent, de faible épaisseur, a été découpé et déposé sur la suspension pendant 15 à 25 min. Les oocystes se sont collés au plastique flottant. Ce dernier a été enlevé et rincé avec du sérum physiologique dans une boîte de Pétri. Cette opération répétée a permis d'avoir un grand nombre d'oocystes dans un volume de sérum physiologique relativement important. La solution ainsi obtenue a été centrifugée. Le surnageant a été jeté tandis que le culot a été dilué dans le sérum physiologique. Cette opération, appelée lavage des oocystes, a été 
répétée une fois et 3 à 5 gouttes de bicarbonate de potassium à 1 p. 100 ont été additionnées à la solution concentrée des oocystes. Environ $10^{6}$ oocystes ont ainsi été concentrés dans $50 \mathrm{ml}$ de sérum physiologique.

\section{Incubation des oocystes}

Les oocystes ont été incubés à l'étuve à la température de $27^{\circ} \mathrm{C}$ pendant cinq jours. Quatre-vingt-dix-huit pour-cent des oocystes ont sporulé.

\section{Identification des espèces}

L'identification des coccidies de caprins a été basée sur la couleur et sur la morphologie des oocystes et des sporocystes, en particulier les mensurations des oocystes. Des gouttes de suspension d'oocystes ont été montées entre lame et lamelle et observées au microscope photonique. Les oocystes ont été mesurés (les mensurations ont été effectuées sur, au moins, 100 oocystes par espèce), photographiés et dessinés. Les caractères, tels que la couleur des oocystes et l'absence ou la présence des résidus oocystiques et sporocystiques, ont été directement remarqués. Les auteurs ont identifié les espèces en les comparant aux descriptions fournies par de nombreux auteurs $(1,4,5,9-12,15,16,18-22)$.

\section{Microscopie photonique sur organe post-mortem}

Des fragments des différentes parties de l'intestin ont été fixés au Carnoy, déshydratés à l'éthanol et inclus dans de la paraffine. Des coupes de 6 à $7 \mu \mathrm{m}$ d'épaisseur ont été réalisées à l'aide d'un microtome et colorées, après déparaffinage et hydratation, à l'hématoxiline-éosine ou au trichrome de Masson.

\section{Microscopie électronique à transmission sur organe post-mortem}

Des fragments de l'intestin parasité ont été fixés pendant $1 \mathrm{~h}$ au glutaraldéhyde à 2,5 p. 100 dans le tampon cacodylate de sodium $0,1 \mathrm{M}$ à $\mathrm{pH}$ 7,2 puis pendant $1 \mathrm{~h}$ avec du tétroxyde d'osmium à 1 p. 100 dans le même tampon. Ils ont ensuite été déshydratés à l'éthanol et à l'oxyde de propylène avant d'être inclus dans de l'Epon. Les coupes ont été réalisées à l'ultramicrotome Porterblum MT1, puis contrastées avec de l'acétate d'uranyle et du citrate de plomb. Elles ont été observées avec un microscope électronique JEOL100 C X II.

\section{Microscopie électronique à balayage sur organe post-mortem}

Les procédés de fixation étaient les mêmes qu'en microscopie électronique à transmission. La déshydratation a été faite à l'acétone. Les pièces ont ensuite été passées au point critique, puis recouvertes d'une fine couche d'or et observées au microscope JEOL 35 CF.

\section{Infestations expérimentales}

Un chevreau mâle âgé de huit mois environ et pesant $11 \mathrm{~kg}$ a été utilisé. Il était apparemment sain car les examens coprologiques n'ont pas révélé la présence de parasites gastro-intestinaux. Il a néanmoins été soumis aux traitements suivants pendant la période d'adaptation de deux semaines :

- pénicilline 1 million UI : 0,2 ml par jour pendant cinq jours en injection intramusculaire ;
- vaccin anti-pasteurellique : $12 \mathrm{ml}$ en injection sous-cutanée ;

- nivaquine distribuée à la dose de $150 \mathrm{mg}$ par $10 \mathrm{~kg}$ du poids vif pendant cinq jours.

Le chevreau a été nourri à la paille d'arachide.

A l'issue de ces traitements, de nouveaux examens coprologiques ont été réalisés mais n'ont donné que des résultats négatifs. Le chevreau a été ensuite infesté par voie orale avec environ $6.10^{5}$ oocystes.

\section{Autopsie}

L'animal a été autopsié immédiatement après sa mort qui est intervenue 19 jours après l'infestation. Des fragments d'intestin ont été prélevés pour des études histopathologiques.

\section{RESULTATS}

\section{Taxonomie}

Huit espèces de coccidies du genre Eimeria ont été trouvées dans les matières fécales de chèvres récoltées aux abattoirs de Dakar. Sept de ces huit espèces étaient connues : Eimeria hirci Chevalier, 1966 (= E. crandallis) (figures 1, 9) ; Eimeria arloingi (Marotel, 1905) Martin, 1909 (= E. bakuensis, E. ovina) (figures 2, 10); Eimeria christenseni Levine, Ivens et Fritz, 1962 (figures 3, 11) ; Eimeria alijevi Musaev, 1970 (= E. parva) (figures 4, 12); Eimeria ninakohlyakimovae Yakimoff et Rastegaieff, 1930 (= E. ovinoidalis) (figures 5, 13) ; Eimeria caprovina Lima, 1980 (figures 6, 14) et Eimeria kocharli Musaev, 1970 (= E. intricata) (figures 7, 15).

La huitième espèce était inédite ; Elle a été décrite, ci-dessous, par les auteurs sous le nom de :

\section{Eimeria panguii n. sp. (figures 8,16 )}

Les oocystes sporulés étaient ovoïdes et de grande taille. Ils mesuraient 48,19 $\pm 0,73 \mu \mathrm{m}$ de long sur $32,45 \pm 0,97 \mu \mathrm{m}$ de large (45-50 $\mu \mathrm{m}$ x 29-36 $\mu \mathrm{m})$. Le rapport longueur-largeur des oocystes était de 1,48. Le micropyle était étroit. La capsule micropylaire était absente. La paroi était lisse, épaisse et paraissait constituée de deux couches claires séparées par une couche brune. Cinq résidus oocystiques ont été observés. Les sporocystes étaient ovoïdes et mesuraient $16,86 \pm 0,77 \mu \mathrm{m}$ de long sur $8,93 \pm 0,34 \mu \mathrm{m}$ de large (15-18 $\mu \mathrm{m} \times 8-10 \mu \mathrm{m})$. Le rapport longueur-largeur des sporocystes était de 1,88 . Ils ne présentaient pas de corps de Stieda et contenaient quelques résidus sporocystiques. A maturité, chaque sporocyste contenait deux sporozoïtes falciformes inversés l'un par rapport à l'autre qui se recouvraient par leurs deux extrémités.

\section{Infestation expérimentale et histopathologie}

Quinze jours après l'infestation, une abondante diarrhée a été observée. De cette diarrhée, $33 \times 10^{6}$ oocystes ont été concentrés dans $85 \mathrm{ml}$ de sérum physiologique. Ils ont été incubés à $27{ }^{\circ} \mathrm{C}$ pendant cinq jours. Environ 35 p. 100 de ces oocystes ont sporulé.

Les huit espèces de coccidies trouvées dans les fèces des chèvres abattues aux abattoirs ont été retrouvées après l'infestation du chevreau et leur pourcentage a été estimé : Eimeria hirci (0,7 p. 100), Eimeria arloingi (1 p. 100), Eimeria alijevi (32 p. 100), Eimeria ninakohlyakimovae (51 p. 100), Eimeria caprovina (9 p. 100), Eimeria kocharli (0,3 p. 100), Eimeria christenseni 3,8 p. 100 et Eimeria panguii (2,2 p. 100). 

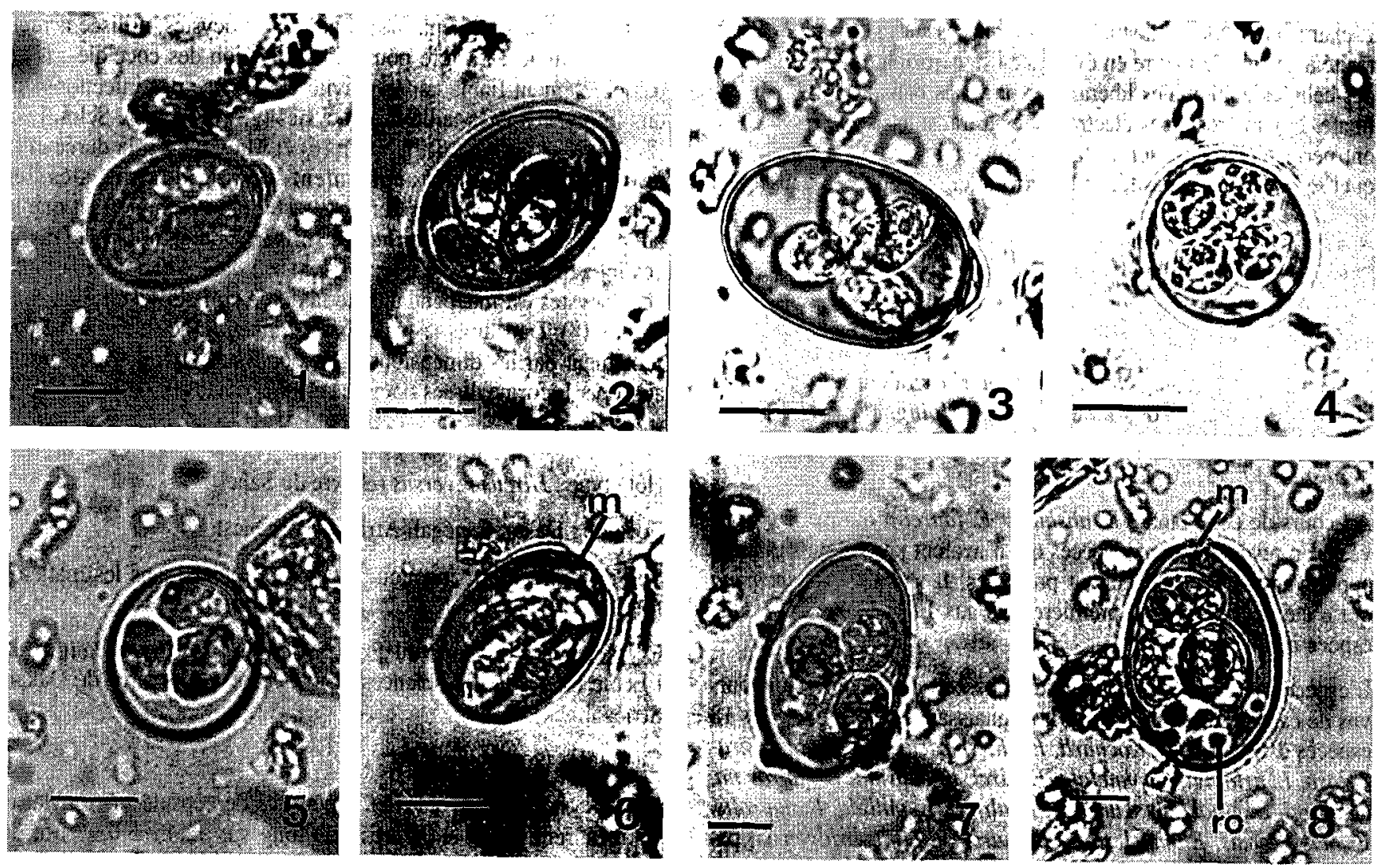

Figure 1 : oocysle $d^{\prime}$ Eimeria hirci (barre $\left.=10 \mu \mathrm{m}\right)$.

Figure 2 : oocyste d'Eimeria arloingi (barre $=10 \mu \mathrm{m}$ ).

Figure 3 : oocyste $d^{\prime}$ Eimeria christenseni (barre $\left.=10 \mu \mathrm{m}\right)$.

Figure 4: oocyste $d^{\prime}$ Eimeria alijevi (barre $\left.=10 \mu \mathrm{m}\right)$.

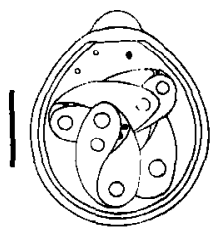

9

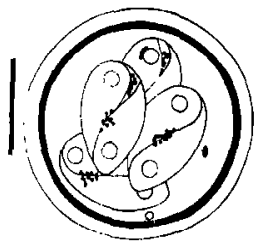

12

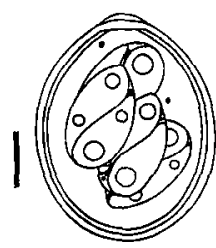

10

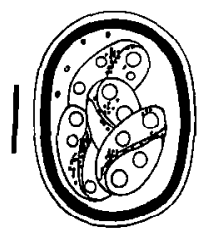

13

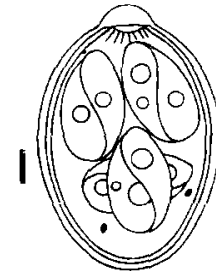

11

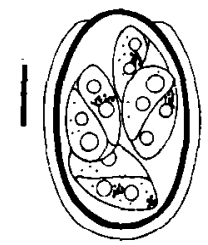

14

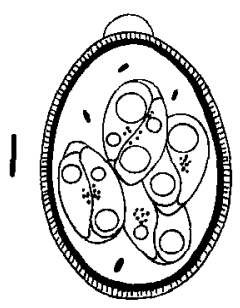

15

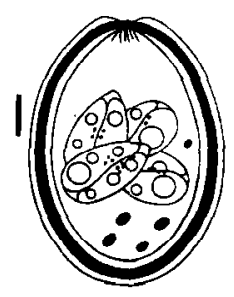

16
Figure 5 : oocyste d'Eimeria ninakohlyakimovae (barre $=10 \mu \mathrm{m})$.

Figure 6: oocyste d'Eimeria caprovina (barre $=10 \mu \mathrm{m})$.

Figure 7 : oocyste $d^{\prime}$ Eimeria kocharli (barre $\left.=10 \mu \mathrm{m}\right)$.

Figure 8 : oocyste $d^{\prime}$ Eimeria panguii (barre $\left.=10 \mu \mathrm{m}\right)$.

Figures 9 à 16 : représentations schématiques des oocystes des coccidies trouvées (barres $=8 \mu \mathrm{m}$ ).

Figure 9 : E. hirci.

Figure $10:$ E. arloingi.

Figure 11 : E. christenseni.

Figure 12 : E. alijevi.

Figure 13 : E. ninakohlyakimovae.

Figure 14 : E. caprovina.

Figure 15 : E. kocharli.

Figure 16 : E. panguii. 
L'examen des coupes histologiques réalisées sur le tube digestif parasité a permis de mettre en évidence l'hypertrophie et l'éclatement des cellules épithéliales libérant les oocystes dans la lumière intestinale. Les microscopes électroniques à transmission et à balayage ont permis aussi d'observer la chute des microvillosités de la muqueuse intestinale parasitée et la perforation de cette muqueuse.

\section{DISCUSSION}

\section{Taxonomie}

Au Sénégal, quelques espèces d'Eimeria avaient été signalées chez des chèvres et des moutons : E. arloingi, E. christenseni, E. alijevi, E. ninakohlyakimovae, E. hirci, E. kocharli, E. faurei et E. ahsata $(17,18,20,21,22)$.

Au cours de cette étude, E. ahsata et E. faurei n'ont pas été trouvées. En revanche, deux espèces qui n'avaient jamais été signalées auparavant au Sénégal étaient présentes. Il s'agit d'E. caprovina qui a été décrite pour la première fois aux Etats-Unis (12) et une espèce inédite.

L'espèce inédite, décrite ci-dessus, possède des oocystes dépourvus de capsule micropylaire. A la connaissance des auteurs, des 18 espèces d'Eimeria (E. Kocharli, E. christenseni, E. ahsata, E. granulosa, E. arloingi, E. punctata, E. hirci, E. caprina, E. caprovina, E. faurei, E. ninakohlyakimovae, E. alijevi, E. pallida, E. capralis, E. masseyensis, E. charlestoni, E. marsica, E. weybridgensis) parasites du mouton et de la chèvre, décrites à travers le monde par de nombreux auteurs $(1,4,5,11,12,15,16,18,19)$, sept produisent des oocystes également dépourvus de capsule micropylaire. Ce sont : E. caprina, E. caprovina, E. faurei, E. ninakohlyakimovae, E. alijevi, E. pallida et E. charlestoni $(9,11,12,15,16)$. La comparaison de ces sept espèces à la présente espèce permet de constater que :

- E. caprina, E. caprovina et $E$. faurei possèdent des oocystes dont la longueur correspond aux deux tiers de celle des oocystes de l'espèce décrite ;

- E. ninakohlyakimovae et E. charlestoni ont des oocystes dont la longueur correspond à la moitié de celle des oocystes de la présente espèce ;

- la longueur des oocystes d'E. alijevi et d'E. pallida correspond au tiers de celle des oocystes de la présente espèce ;

- les oocystes d'E. alijevi, d'E. ninakohlyakimovae, d'E. caprina, d'E. caprovina, d'E. pallida et d'E. charlestoni se distinguent des oocystes de la présente espèce par leur forme qui peut être ellipsoïde, sphérique ou subsphérique ;

- les oocystes d'E. faurei possède un micropyle étroit associé à un bouton intraoocystique (21);

- les oocystes d'E. caprovina, d'E. charlestoni et d'E. caprina possèdent un micropyle plus large que celui des oocystes de la présente espèce $(11,12,16)$;

- la longueur des sporocystes des sept espèces est nettement plus petite que celle des sporocystes de l'espèce décrite ici ;

- les sporocystes d'E. charlestoni, d'E. ninakohlyakimovae, d'E. caprovina et d'E. caprina se distinguent de ceux de l'espèce inédite par la présence du corps de Stieda.

Tous ces éléments distinctifs ont amené les auteurs à penser qu'ils étaient en présence d'une espèce nouvelle qu'ils proposent d'appeler Eimeria panguii n. sp., en hommage au Professeur Louis Pangui, Parasitologiste à l'Ecole inter état des sciences et médecine vétérinaires de Dakar, Sénégal.
Certains auteurs pensent que la taille des oocystes, utilisée actuellement comme caractère pour l'identification des coccidies, n'est pas un élément fiable car la gravité et la durée des infections ont une influence sur la taille des oocystes $(2,3,6,7,13)$. Selon Fish (7), Beker et coll. (2, 3), Duncan (6) et Majaro (13), la durée et la gravité des infections augmentent la taille des oocystes des Eimeria. D'autres auteurs pensent le contraire et rapportent que la taille des oocystes n'est influencée ni par la durée ni par la gravité des infections (8). C'est le cas d'Eimeria acervulina dont la taille des oocystes est indépendante de la durée et de la sévérité de l'infection (8). Dans tous les cas, Eimeria panguii ne se distingue pas seulement par les dimensions de ses oocystes et sporocystes mais aussi par la forme de ses oocystes et sporocystes.

\section{Diagnose d'Eimeria panguii $n$. sp.}

Hôte type : Hircus reversus (chèvre du Sahel).

Localité : Dakar, Sénégal, Afrique de l'Ouest.

Site de l'infection : inconnu ; oocystes trouvés dans les matières fécales.

Etymologie : du nom du Professeur Louis Joseph Pangui de l'Ecole inter état des sciences et médecine vétérinaires de Dakar, Sénégal.

\section{Caractères morphologiques :}

Les oocystes sont ovoïdes et dépourvus de capsule micropylaire. Ils mesurent 48,19 $\pm 0,73 \mu \mathrm{m}$ de long sur $32,45 \pm 0,97 \mu \mathrm{m}$ de large (45-50 $\mu \mathrm{m}$ x 29-36 $\mu \mathrm{m})$. Le micropyle est étroit. Les résidus oocystiques sont présents. Les sporocystes sont ovoïdes et mesurent $16,86 \pm 0,77 \mu \mathrm{m}$ de long sur 8,93 $\pm 0,34 \mu \mathrm{m}$ de large $(15-18 \mu \mathrm{m} \mathrm{x}$ 8-10 $\mu \mathrm{m})$. Le corps de Stieda est absent tandis que les résidus sporocystiques sont présents. Les deux sporozoïtes sont falciformes et inversés l'un par rapport à l'autre et se recouvrent par leurs deux extrémités.

\section{Histopathologie}

De nombreux travaux avaient déjà montré que les coccidies intestinales de mammifères causent chez leurs hôtes la perforation, la lyse et la desquamation de la muqueuse intestinale ainsi que des ulcérations qui peuvent, dans des cas de fortes infestations, provoquer leur mort $(14,15)$.

\section{BIBLIO GRAPHIE}

1. BARUTZKI D., GOTHE R., 1988. Zur Kokzidienfauna der Schafe: Artdifferenzierung der 0 ozysten. Weiner Tierärztl. Monatsschr., 12: 494498.

2. BECKER E.R., JESSEN R.J., WATER H.P., VANDOORNINK W.A., 1956. A Biometric study of the oocyst of Eimeria necatrix: a parasite of common fowl. J. Protozool., 3: 126-131.

3. BECKER E.R., ZIMMERMANN W.J., PATILLO W.H., 1955. A biometric study of the oocyst of Eimeria brunetti, a parasite of the common fowl. J. Protozool., 2: 145-150.

4. CHEVALIER H.J., 1986. Uber die Coccidienarten der Ziegen in Deutschland. Dtsch. Tierarztl. Wochenschr., 73: 616-621.

5. CHHABRA R.C., PANDEY V.S., 1991. Coccidia of goats in Zimbabwe. Vet. Pathol., 39: 199-205.

6. DUNCAN S., 1959. The size of the oocysts of Eimeria labbeana. J. Parasitol., 45: 191.

7. FISH F.F., 1931. Q ualitative and statistical analysis of infections with Eimeria tenella in chickens. Am. J. Hyg., 14: 560.

8. JONES E.E., 1932. Size as a species characteristic in coccidia. Variation under diverse conditions of infection. Arch. Protistenk., 76: 130. 
9. LEVINE N.D., IVENS I., 1986. The coccidian parasites (Protozoa, A picomplexa) of Artiodactyla. Illinois Biol. Monogr., 55: 1-265.

10. LEVINE N.D., IVENS I., FRITZ T.E., 1962. Eimeria christenseni sp. n. and other coccidia (Protozoa: Eimeridae) of the goat. J. Protozool., 48: 255-269.

11. LIMA D.J., 1979. Eimeria caprina sp. n. from the domestic goat Capra hircus, from the USA. J. Protozool., 26: 902-903.

12. LIMA D.J., 1980. Eimeria caprovina sp. n. from the domestic goat, Capra hircus, from the USA. J. Protozool., 27: 153-154.

13. MAJARO O.M., 1988. Effects of severity and duration of infections on oocyst size of Eimeria necatrix, a coccidium of chiken. Revue Elev. Méd. vét. Pays trop., 41: 167-170.

14. OPOKU-PARE G.A., CHINENE C.N., 1979. Pathologie de la coccidiose intestinale aigüe chez les jeunes caprins. Bull. Santé Prod. Anim. Afr., 27 : 295-304.

15. SHAH H.L., JOSHI S.C., 1963. Coccidia (Protozoa: Eimeriidae) of goats in Madhya Pradesh, with descriptions of the sporulated oocysts of eight species. J. vet. Anim. Husb. Res., 7: 9-20.

\section{Summary}

Maho A., Toguebaye B.S., Belot J. Intestinal coccidia and coccidioses of the Sahel Goat (Hircus reversus) in Senegal with the description of a new species

Eight Eimeria species, of which one of them was new, were found in Sahel goats (Hircus reversus) from Senegal: E. arloingi, E. kocharli, E. alijevi, E. ninakohlyakimovae, E. christenseni, E. hirci, E. caprovina, and Eimeria panguii n. sp. O ocysts of Eimeria panguii n. sp. were ovoid, without a micropylar cap and measured $48.19 \pm 0.73 \times 32.45 \pm 0.97 \mu \mathrm{m}$ (45-50 x 29-36 $\mu \mathrm{m}$ ). The micropyl was small. O ocyst residua were present. Sporocysts were ovoid, $16.86 \pm 0.77 \times 8.93 \pm 0.34 \mu \mathrm{m}(15-18 \times 8-10 \mu \mathrm{m})$. The Stieda body was absent, whereas sporocyst residua were present. Both sporozoites were sickle-shaped, in an inverted position with each other and overlapped each other from both ends. Experimental transmission of these species to a kid revealed thicknening of the gut epithelial cells, perforation of the intestine mucosa and loss of its microvilli.

Key words: Sahel Goat - Coccidia - Coccidiosis - Eimeria panguii n. sp. - New species - Senegal.
16. SOE A.K., POMROY W.E., 1992. N ew species of Eimeria (Apicomplexa: Eimeriidae) from the domesticated goat Capra hircus in N ew Zealand. Syst. Parasitol., 23: 195-202.

17. TOUMBA G., 1989. La coccidiose intestinale chez la chèvre du Sahel : observation clinique et histopathologique après l'infestation expérimentale. Thèse doct., U niversité de Dakar, Dakar, Sénégal, $97 \mathrm{p}$.

18. VASSILIADES G., 1965. Sur un foyer de coccidiose intestinale du mouton dans la presqu'lle du Cap Vert à Sébikotane, Sénégal. Revue Elev. Méd. vét. Pays trop., 18 : 145-149.

19. VASSILIADES G., 1969. La coccidiose intestinale des ruminants domestiques au Sénégal - Epidémiologie, répartition géographique, importance économique. Revue Elev. Méd. vét. Pays trop., 22 : 47-53.

20. VASSILIADES G., 1981. Parasitisme gastro-intestinal chez le mouton du Sénégal. Revue Elev. Méd. vét. Pays trop., 34 : 169-177.

21. VERCRUYSSE J., 1982. The coccidia of sheep and goats in Senegal. Vet. Parasitol., 10: 297-306.

22. YVORE P., ESNAULT A., 1984. Les coccidies des ruminants. Diagnoses d'espèces. Bull. G.T.V., 6 : 13-18.

Reçu le 27.2.96, accepté le 16.9.97

\section{Resumen}

Maho A., Toguebaye B.S., Belot J. Coccidias y coccidiosis intestinales de la cabra del Sahel (H ircus reversus) en Senegal, con la descripción de una nueva especie

Se encontraron ocho especies de Eimeria, incluyendo una nueva especie, en la cabra del Sahel (Hircus reversus) en Senegal. Son las siguientes: E. arloingi, E. kocharli, E. alijevi, E. ninakohlyakimovae, E. christenseni, E. hirci, E. caprovina y Eimeria panguii n. sp. Los oocitos de Eimeria panguii n. sp. son ovoides y desprovistos de cápsula micropilar. Miden $48,19 \pm 0,73 \times 32,45 \pm 0,97 \mu \mathrm{m}(45-50 \times 29-36 \mu \mathrm{m})$. El micrópilo es estrecho. Los residuos oocísticos estan presentes. Los esporocitos son ovoides, $16,86 \pm 0,77 \times 8,93 \pm 0,34 \mu \mathrm{m}$ (15-18 x 8-10 $\mu \mathrm{m})$. Los cuerpos de Stieda están ausentes, mientras que los residuos esporocísticos están presentes. Los dos esporozoitos son falciformes e invertidos entre ellos y se cubren en las dos extremidades. La transmisión experimental de estas especies a un cabrito, permitió demostrar la hipertrofia de las células del epitelio intestinal, la perforación de la mucosa intestinal y la caída de las microvellosidades.

Palabras clave: Caprino de Sahel - Coccidia - Coccidiosis Eimeria panguii n. sp. - Especie nueva - Senegal. 